\title{
Antenna Aperture Synthesis For SFCW GPR in a Medium with Frequency Dispersion of Radio-Waves Propagation Phase Velocity
}

\author{
Volodymyr G. Sugak \\ Department of Statistical Radiophysics, \\ Institute of Radiophysics and Electronic NASU \\ Kharkiv, Ukraine \\ vladymyr.sgk@gmail.com
}

\author{
Alexander Dubovitsky \\ Research Center \\ Belgorod National Research University \\ Belgorod, Russia
}

\begin{abstract}
The problem of antenna aperture synthesis has been solved for the georadar probing on a relatively large surface area of the ground to increase the resolution in the horizontal plane in depth. Aperture synthesis is carried out with a priori unknown values of the radio wave propagation phase velocity below the surface of the soil and its frequency dispersion. For this, iterative procedures are used, in which the radio wave deceleration factor in the soil and the form of the frequency dependence of the radio wave propagation phase velocity are changed. Iterative procedures are completed when the image contrast of the heterogeneities of the subsurface soil structure reaches the greatest value.
\end{abstract}

Index Terms-Georadar; antenna aperture synthesis; iterative technique

\section{INTRODUCTION}

In modern conditions, Ground Penetrating Radar (GPR), or georadar technologies are often used in such areas like the engineering geology, construction geology and ecology. At the same time, typical tasks of such applications are to detect rather small buried objects including inhomogeneities of both artificial and natural character at the limiting depths of the zone up to several tens of meters $[1,2,4,5]$. In this case, it is necessary to have a depth resolution of about tens of centimeters, which requires the use of a spectrum of probing signals of at least some hundred megahertz. The frequency dispersion of the electrical characteristics of the soil leads to a greater attenuation of the high-frequency components of the probe signal and to distortions of its phase structure due to the functional dependence of the radio wave propagation phase velocity on the frequency in the soil. However, the most limiting factor is the insufficiently resolving power of the method in the horizontal plane at the required depths, which is determined by the relatively wide georadar antenna directional pattern.

The standard technique of probing and signal processing does not allow obtaining the required resolving power of the probing signal in the horizontal plane on depth.

This work is devoted to the evaluation of the efficiency of using the method of georadar antenna aperture synthesize when probing over large areas, the size of which is up to the size of a georadar antenna. At each probing point, the coordinates of which are considered exactly known, the probing and reflected signals are stored in the computer memory. After accumulating the information about the reflected signals at all the probing points one can execute their processing, one of the stages of which is the antenna aperture synthesis of the corresponding to the area where the measurements were made. The possibility of synthesis is conditioned the quadrature components obtaining at the output of the GPR receiver, that is, their phase structure.

As a probing, we consider a Stepped Frequency Continuous Wave (SFCW) signal, which, as shown in [4,5], makes it possible to correct the above mentioned distortions and to increase the contrast of images when processing the signals reflected by subsurface inhomogeneous objects.

\section{FEATURES OF REFLECTION OF RADIO-WAVE FROM OBJECTS LOCATED UNDER THE EARTH SURFACE}

Consider an object located at a depth of $d$ meters, irradiated by a component of the electric field.

The SFCW GPR determines the distance to the object, processing radar reflections in a coherent mode on each of the successively varying carrier-frequency values.

The electrical component $E_{x}^{r}(\omega)$ is then converted into a voltage on the receiver's input. Then, in a coherent receiver, the signal is shifted down the range using a quadrature demodulator to obtain the quadrature (Im and Re) components of the received signal [6].

The method for compensating for signal distortions caused by the frequency dependence of the radio waves propagation phase velocity in a dispersive medium is based on the ability of revealing the functional dependence of the electrical characteristics of the soil upon the frequency, and, consequently, the dependence of the phase velocity of radio waves in soil layers directly from the results of radar subsurface probing. The fundamentals of the algorithm for reconstructing this functional dependence are presented in [7]. 


\section{III.AlgORITHM OF THE ANTENNA AERTURE SYNTHESIS AT COMPENSATION OF PHASE Distortions OF REFLECTED SIGNALS}

Initially, the electrical characteristics of the medium are set, representing the functional dependences of the permittivity and conductivity of the selected soil type upon the frequency. These dependencies are determined on the basis of the model, in which it is possible to specify the type of soil, moisture, degree of mineralization, etc. This model is described in details in [5].

In Fig. 2, as an example, there are shown two functional dependences of the deceleration factor of the radio wave propagation phase velocity in the loam upon the frequency for the values of volume moisture of $12 \%$ (curve 1) and $18 \%$ (curve 2).

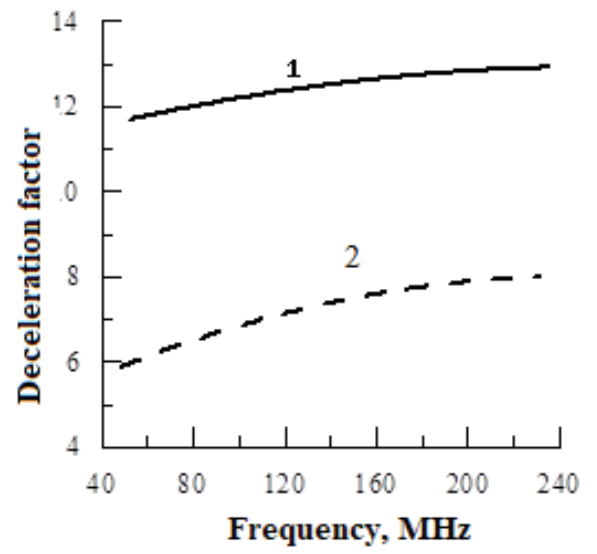

Fig. 2. Functional dependences of the radio waves propagation phase velocity in the loam upon the frequency

Such functional dependencies are easily approximated by a quadratic polynomial in a given frequency range, the coefficients of which can be determined from the above mentioned model of the dependence of the electrical characteristics of a considered soil type upon the frequency. The function, which is inverse to the approximating quadratic polynomial can be used as the compensating factor in the expression for the received signal at the output of the receiver of a SFCW GPR.

In literature, there is no sufficient information on the technique for the antenna aperture synthesis for georadar probing. Some useful information can be found in [6].

When synthesizing the aperture of the antenna, one must take into account the fact that not at all the probing points the georadar antenna will irradiate the selected resolution element due to the finite width of the radiation pattern. Therefore, it is necessary to use a sliding window, the size of which (the size of the antenna aperture) should be selected based on the above information. As a result, the complex synthesized signal corresponding to the $q$-nth point of the sub-surface space is obtained by using a time delay and its weighting with a special function.

$$
z_{q, n}(t)=\sum_{r, m} w_{r n} a\left(\vec{x}_{q}\right) s\left(t-\tilde{\tau}_{m n}\right)
$$

where $\tilde{\tau}_{m n}$ is the focusing delay applied to the output signal in the $n$-th position of the receiver and the $m$-th position of the transmitter, while $q$ is the $i$-th space current.

Consider some results of practical application of the described algorithm for synthesizing the antenna aperture when probing along several profiles of the georadar motion.

The probing was performed along the asphalt road. There were used five parallel profiles with a length of $10 \mathrm{~m}$ with a shift of $20 \mathrm{~cm}$ and with the discrete step of $20 \mathrm{~cm}$ that crossed a pipe laid under the surface of the roadway.

It was used a SFCW GPR with the following technical characteristics: the radiated power about $1 \mathrm{~W}$, the operating frequency range $285-430 \mathrm{MHz}$ depending on the structure and humidity of the ground, the resolution in depth from 25 to 35 $\mathrm{cm}$, and the accumulation time from $1.2 \mathrm{~s}$.

In the data processing, the antenna aperture was synthesized using a sliding window whose size was \pm 5 discrete steps relative to each probing point along the motion profile and the \pm 2 steps across the movement. That is, the size of the aperture of the synthesized antenna was approximately $260 \times 140 \mathrm{~cm}$, which is equivalent to the width $\theta_{\|}$of the radiation pattern along the profile of the georadar motion, and $\theta_{\perp}$ across its movement in free space, respectively,

$$
\theta_{\|}=\frac{\lambda}{l}=\frac{1}{2.4} \approx 0.4 \text { radian and } \theta_{\perp}=\frac{\lambda}{l}=\frac{1}{1.4} \approx 0.7 \text { radian }
$$

This window slid over all the probing data corresponding to the selected site. The synthesizing algorithm was based on the following iterative procedures. Initially, the value of the deceleration factor was set, which was certainly greater than the true value. Then, for a given value of the deceleration factor, iterations were performed in which the form of the frequency dependence of the phase velocity of propagation is changed. Then, the value of the deceleration factor of the radio wave propagation phase velocity was decreased by some discrete value and the iteration process was repeated again.

At each iteration, a synthesized image of the radar section of the soil along the selected profile was constructed. As a result, the image was selected in which the greatest contrast of the object under study was observed. In the future, it will be possible to develop a fully automatic procedure for stopping the iterations to achieve the maximum value of the signal-tointerference ratio.

One important fact should be noted. When the image is focused after completing the iterations, the real values of the deceleration factor and the form of the frequency dependence of the radio wave propagation phase velocity are obtained.

In addition, in the final radar image of the section of the ground, the detectable objects are already located at depths close to their true values.

In Figs. 3 and 4, we show the results of the antenna aperture synthesis when probing at a selected site.

Fig. 3 corresponds to a faintly focused radar image of the section of the ground, which corresponds to the size of the sliding window of the synthesized aperture, equal to $1 \mathrm{x} 1 \mathrm{~m}^{2}$. It can be seen that with this size of the antenna aperture the 


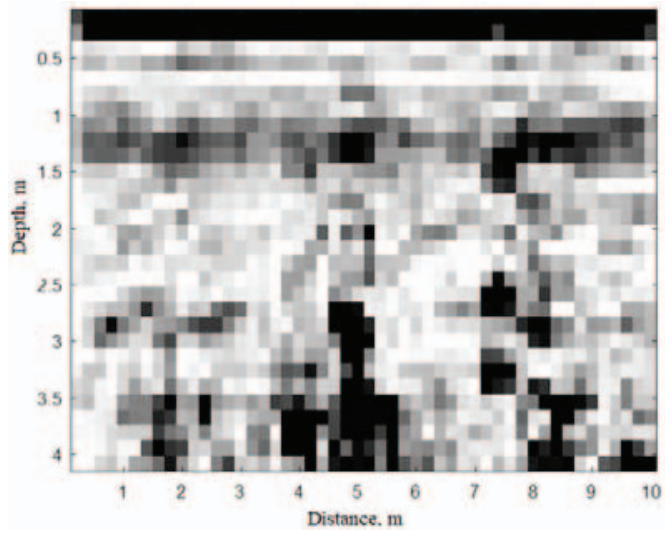

Fig. 3. Synthesized radar image of the cross section of the ground along the profile of the georadar movement, crossing the direction of the water pipe at a sliding aperture window $1 \times 1 \mathrm{~m}$

image is very blurry, and the signal to noise ratio does not allow revealing confidently the reflected from object signal.

It should be noted that on a standard image of the section of the soil along any of the parallel profiles of the georadar motion, the signal reflected from the investigated object was completely masked by reflections from the heterogeneities of the soil structure.

The next image (Figure 4) corresponds to the focused image, which was obtained by using a sliding window with a size of $2.6 \times 1.4 \mathrm{~m}$ and a value of the propagation velocity decay factor of 0.085 . The image corresponds to one of the five GPR profiles.

It can be seen that the image of the object under investigating object (the water pipe) has already a good contrast, and the signal-to-noise ratio is sufficient for assured detection of signal reflected from this object. To confirm this, Fig. 5 shows the amplitudes of the signals reflected from the inhomogeneities of the subsurface structure of the soil, including signals from the object under study. It can be seen that the signal-to-noise ratio at the location of the object is approximately of $15 \mathrm{~dB}$.

\section{CONCLUSIONS}

A method for synthesizing the antenna aperture has been proposed for georadar probing on a site whose dimensions are substantially larger than the size of the georadar antenna. This allows one to gain in resolving power from angular coordinates. The method is based on the correction of phase distortions of radar signals reflected by subsurface ground heterogeneities, applied to a SFCW probing signal and iterative procedures in which the form of the correction coefficient changes, the functional frequency dependence of which is inverse to the frequency dependence of the phase distortions.

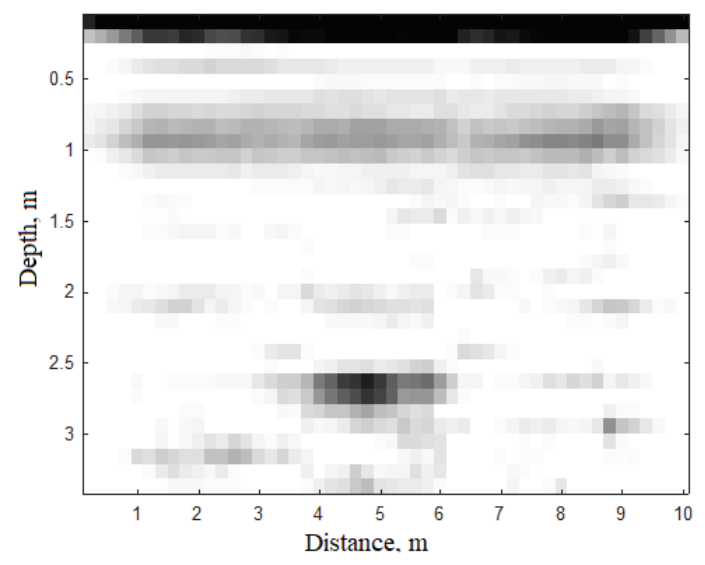

Fig. 4. The focused radar image of the cross section of the ground along the profile of the georadar movement, crossing the direction of the pipe

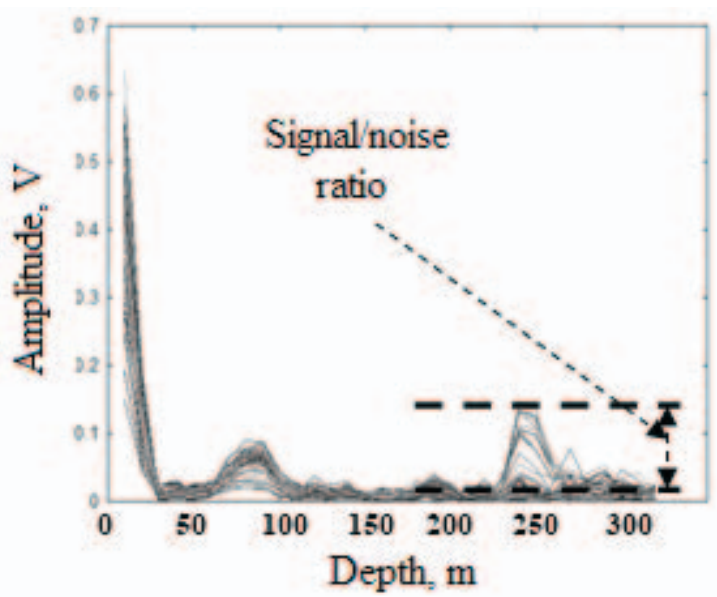

Fig. 5. The amplitudes of the signals reflected by inhomogeneities of the subsurface structure along one of the georadar motion profiles

\section{REFERENCES}

[1] L. Peters, J. Danials, and J. Joung, "Ground penetrating radar as a subsurface environmental sensing tool," IEEE Proc., vol. 82, pp. 1803 $1821,1994$.

[2] M. P. Hurst and R. Mittra, "Scattering center analysis via Prony's method," IEEE Trans. Antennas Propagat., vol. 35, no 8, pp. 986-988, 1987.

[3] F. N. Kong and T. L. By. "Performance of a GPR system which uses step frequency signals," J. Appl. Geophys., vol. 33, no 1-3, pp. 15-26, 1995.

[4] V. G. Sugak and A. V. Sugak, "Phase spectrum of signals in ground penetrating radar applications," IEEE Trans. Geoscience Remote Sens., vol. 48, no 4, pp. 1760-1767, 2010.

[5] V. G. Sugak, "Particularities of signal processing at subsurface radar sounding in dispersive media," Telecommunic. Radio Eng., vol .66, no 16, pp. 1425-1440, 2007.

[6] F. Ahmad, G. J. Frazer, S. A. Kassam, M. G. Amin, “A new approach for near-Field Wideband Synthetic Aperture Beamforming,", Proc. IEEE Int. Conf. Acoust., Speech, Signal Process. 2003 (ICASSP-2003), 2003, vol. 5, pp. 89-92.

[7] V. G. Sugak, A. V. Bukin, and A. Djaduei, "Probing signal correlation function in ground penetrating radar applications," Applied Radio Electronics, vol. 14, no 3, pp. 197-203, 2015. 